\title{
SERVICIOS DE REFERENCIA EN BIBLIOTECAS UNIVERSITARIAS: TENDENCIAS Y PLAN DE MARKETING
}

Nieves González-Fernández-Villavicencio

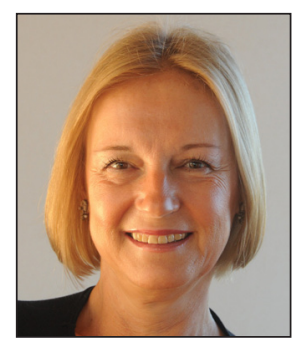

Nieves González-Fernández-Villavicencio es responsable del Área de Biblioteconomía y Documentación de la Universidad Pablo de Olavide (Sevilla) y bibliotecaria de la Universidad de Sevilla, donde ha sido responsable del Servicio de referencia y de Formación en competencias informacionales, impulsando el uso de las herramientas de la web social. Ha sido miembro del grupo de trabajo de las $\mathrm{Cl} 2$ de CRUE/TIC y Rebiun y dirigido el Plan Alba de formación en competencias digitales de la Red de Bibliotecas de la Junta de Andalucía. Imparte cursos y conferencias sobre competencias digitales, servicios de referencia, planificación de servicios bibliotecarios en la web social y social media marketing. Es miembro del Grupo ThinkEPI. http://orcid.org/0000-0001-8940-7429

Universidad Pablo de Olavide, Área de Biblioteconomía y Documentación Ctra. de Utrera, Km. 1, 41013 Sevilla, España nievesglez@gmail.com ngonfer@upo.es

\section{Resumen}

Los servicios presenciales y virtuales de referencia de las bibliotecas universitarias tienen un bajo uso, lo que pone en peligro su continuidad. Bibliotecarios universitarios en todo el mundo están llevando a cabo programas innovadores y creativos para ofrecer el servicio dónde, cómo y cuándo se necesita. Es necesario que las bibliotecas evalúen sus servicios para adaptarse a las necesidades cambiantes de sus usuarios, elaborando e incorporando planes de marketing a sus planes estratégicos que permitan un rediseño del servicio de referencia en función de datos estadísticos, satisfacción de usuarios y ROI. La biblioteca universitaria debe demostrar el valor que aporta a los objetivos de su institución. Se ofrece una visión actual de conjunto del servicio de referencia presencial y virtual de las bibliotecas universitarias, señalando sus errores y aciertos, a partir de una revisión bibliográfica.

\section{Palabras clave}

Servicios de referencia, Bibliotecas universitarias, Servicios virtuales, Rediseño de servicios, Plan de marketing.

\section{Title: Libraries, the promotion of reading and social networks: converting friends into readers}

\begin{abstract}
Reference services, both face-to-face and virtual, in academic libraries have a low usage, which jeopardize their continuity. University librarians worldwide are pursuing innovative and creative programs to offer the service where, how and when it is needed. Libraries have to evaluate their services to meet the changing needs of users, developing and adding marketing plans to strategic plans, which allow a redesign of reference service, depending on statistical data, user satisfaction data, and ROI. The academic library must demonstrate the value it brings to the objectives of the institution. Based on a literature review, an overview of the current face-to-face and virtual reference services in university libraries is provided, pointing their mistakes and successes.
\end{abstract}

\section{Keywords}

Reference services, Academic libraries, Virtual services, Redesign of services, Marketing plan.

González-Fernández-Villavicencio, Nieves. "Servicios de referencia en bibliotecas universitarias: tendencias y plan de marketing". El profesional de la información, 2012, noviembre-diciembre, v. 21, n. 6, pp. 567-576.

http://dx.doi.org/10.3145/epi.2012.nov.03

\section{Introducción}

Uno de los paneles del congreso de la American Library Association (ALA) de junio de 2012 llevaba como lema: "¿Valen la pena los servicios de referencia virtual?". Queremos responder que sí, aunque las estadísticas de uso lleven décadas descendiendo (Logan, 2009), algunos autores consideren que es ineficiente y caro (Luini, 2012), y otros opinen que su 
modalidad virtual es una de las grandes equivocaciones de los bibliotecarios (Coffman, 2012).

http://ala12.scheduler.ala.org/node/1062

En este artículo se ofrece una visión actual de conjunto del servicio de referencia presencial y virtual de las bibliotecas universitarias, señalando sus errores y aciertos a partir de una amplia revisión bibliográfica así como de la experiencia profesional de la autora. Se muestra que este servicio, como cualquier otro de la biblioteca universitaria, debe responder a un plan de marketing y solucionar las auténticas necesidades de los usuarios.

La biblioteca universitaria tiene que estar disponible a cualquier hora y lugar, para cualquier persona y por el mayor número de canales

\section{Descenso en el uso}

El servicio de referencia ofrece asesoramiento e indicaciones sobre creación, gestión, evaluación de recursos de información, herramientas y servicios, para que los usuarios puedan satisfacer sus necesidades de información (RUSA, 2008). La mayoría de bibliotecas tienen estos servicios en modos presencial y virtual o digital (Manso-Rodríguez, 2010) y muy orientados hacia la enseñanza de fuentes de información y técnicas de búsqueda (Merlo-Vega, 2009).

Todos los autores consultados en la bibliografía especializada coinciden en que existe un descenso generalizado de su uso. Basta buscar en Google "decline of reference transactions" para tomar conciencia de esta realidad.

Entre las razones que se aportan en la bibliografía destaca en primer lugar el rápido cambio en la tecnología, que ha modificado la forma en la que los bibliotecarios conciben el servicio de referencia, aunque en muchos casos se beneficien de las nuevas posibilidades que ésta les ofrece (Merlo-

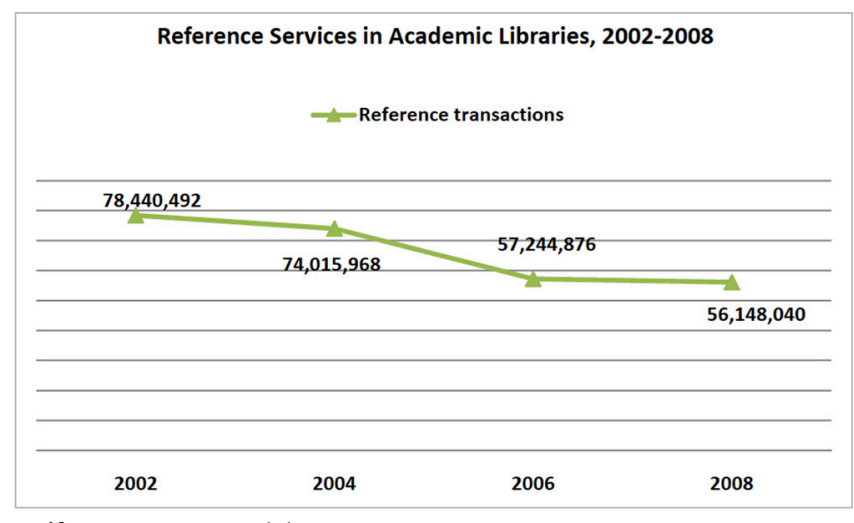

Gráfico 1. Escaso uso del servicio

Vega, 2009). Por otro lado, la proliferación de contenidos en formato digital hace que los usuarios consigan la información en internet de forma fácil y autónoma (Mitchell et al., 2012). Otra razón es el cambio de expectativas de los usuarios en el uso y consumo de información: internet satisface sus necesidades de forma inmediata -al menos aparentemente- y por ello esperan de cualquier servicio que les ofrezca igualmente al instante un acceso al texto completo y (casi exclusivamente) en formato digital, condiciones que las bibliotecas hoy por hoy no satisfacen (Saunders, 2012).

La existencia de competidores con una oferta de servicios sin parangón como Google, Amazon, Facebook, Wikipedia o Twitter (Langan, 2012), hace que las bibliotecas deban competir en inferioridad de condiciones por la atención de un usuario antes cautivo. También la situación económica actual de recortes presupuestarios incide directamente en la disminución de la plantilla y una menor oferta de servicios.

Otras causas internas a la propia biblioteca afectan también a su bajo uso, como pueden ser, en el caso de la referencia virtual, la deficiente identificación y promoción del servicio, que hace que el usuario lo desconozca (Cassell; Hiremath, 2011), o una percepción negativa del mismo cuando éste no le ha sido útil o el bibliotecario no ha sido amable (Connaway; Radford, 2011).

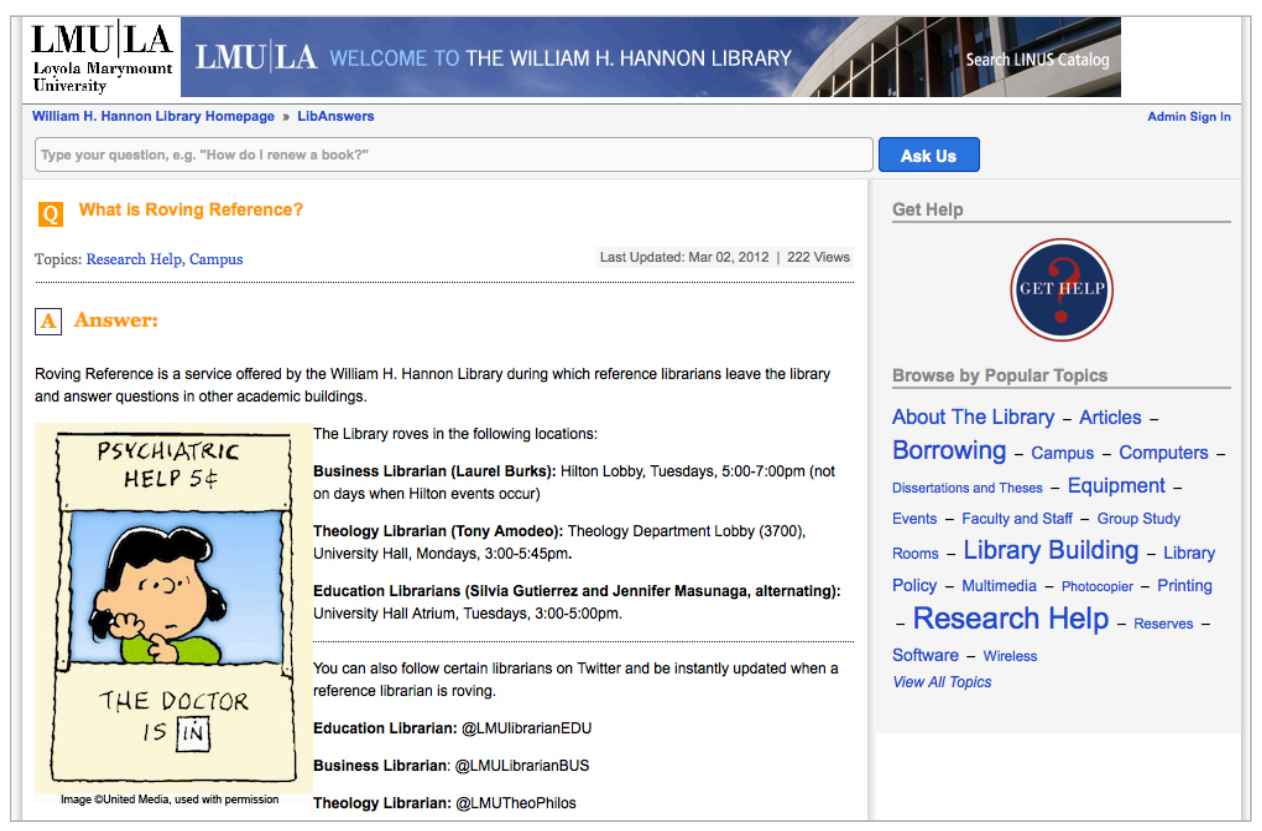

Imagen 1. Roving reference en $L M U$
En este contexto, a partir de la revisión bibliográfica se pueden detectar las siguientes tendencias:

1) En la actualidad la referencia presencial continúa siendo más demandada que la virtual en términos absolutos y constituye la primera opción para la mayoría de usuarios (Ramos; Abrigo, 2012). Saunders (2012) y Mitchell et al. (2012) señalan que estudios en las universidades de Massachusetts y Georgia muestran que los usuarios prefieren la interacción presencial para las transacciones de referencia, a pesar de que la atención personalizada sea escasa. 
2) Sin embargo poco a poco los usuarios van cambiando sus preferencias hacia el acceso remoto (Ward; Phetteplace, 2012) por lo que como respuesta se observa un progresivo aumento de los servicios virtuales.

3) Aunque el número de transacciones desciende, las preguntas que se atienden son de mayor nivel y complejidad (Alexander et al., 2011). En otros casos se constata un aumento en la satisfacción del usuario con el servicio, acom-

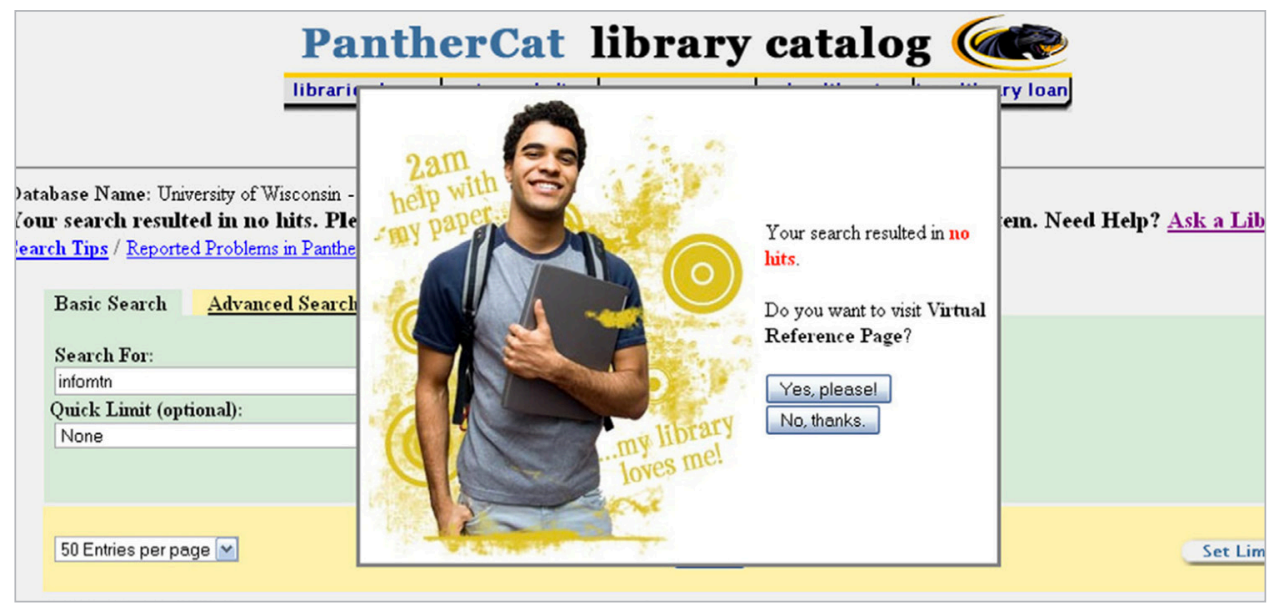

Imagen 2. Pop-up en el catálogo de la Wisconsin University Library pañado de una tendencia a recomendarlo (Mu et al., 2011).

Conscientes de esta situación, muchos bibliotecarios se esfuerzan por mejorar los servicios que ofrecen e innovar con otros, para mantenerse al ritmo de las necesidades cambiantes de sus usuarios. Se puede decir por ello que el servicio de referencia no está en vías de desaparición, sino en pleno desarrollo y evolución (Saunders, 2012).

\section{Oferta de servicios de referencia}

Las formas de referencia han cambiado con el tiempo, desde la entrevista en el despacho o mostrador de referencia, hasta la oferta de servicios virtuales de todo tipo. El mostrador de referencia ya no es necesario como punto de interacción entre bibliotecario y usuario. Los servicios siguen siendo necesarios pero los espacios presenciales ya no (Merlo-Vega, 2009). La innovación se encuentra marcada por cuatro tendencias:

- salir de los espacios físicos habituales;

- oferta de servicios virtuales y móviles;

- uso de herramientas de la web social;

- colaboración entre profesionales.

\section{Servicios presenciales}

Los mostradores de referencia están experimentando una gran transformación, siendo eliminados en algunos casos (Bell, 2007), o fusionados con el de circulación (Alexander et al., 2011). A veces se han integrado con el servicio de soporte TIC (Wang; Henson, 2011), para aprovechar mejor la experiencia de ambos profesionales, con la consiguiente reducción de costes y ofreciendo un modelo de biblioteca universitaria tipo crai -centro de recursos para el aprendizaje y la investigación- (Attis et al., 2011).

Cuando se ha mantenido el espacio o mostrador, se han transformado la señalización y ambiente para destacar los espacios físicos dedicados a la actividad de referencia (Alexander et al., 2011).

En algunos casos se ha sustituido la referencia presencial por un nuevo modelo de servicio bajo demanda, con cita previa con el bibliotecario, y apoyado en un fuerte plan de marketing para que los estudiantes sepan a quién tienen que dirigirse si necesitan ayuda (Arndt, 2010). En estos casos se fortalece el servicio con un equipo de estudiantes a los que

se forma para que puedan responder adecuadamente a las preguntas de bajo nivel (Attis et al., 2011). Esta solución ha recibido críticas por su falta de profesionalidad (debate en el foro Libref-I). Una de las tendencias más consolidadas es la figura emergente del bibliotecario temático vinculado a los departamentos universitarios (Schulte, 2011; Dempsey, 2011).

Dentro de la tendencia señalada de salir fuera de los muros de la biblioteca se debe destacar la roving reference o referencia en movilidad. El bibliotecario abandona los espacios habituales de la biblioteca para satisfacer las preguntas de los usuarios donde éstos se encuentren: en el bar, espacios de estudio, zonas de paso, recintos habituales de los estudiantes, y en los eventos que se organicen en el campus. La telefonía y los dispositivos móviles en general han aumentado las posibilidades de esta opción (Penner, 2011), mediante el uso de iPad (Gadsby; Qian, 2012), portátiles y teléfonos móviles (Henry et al., 2012). Es interesante destacar el caso de los bibliotecarios en movilidad de la Loyola Marymount University de Los Ángeles, por el amplio uso de las herramientas sociales en su trabajo de referencia: Foursquare para hacer checking del lugar en el que están e informar así a los usuarios, sincronizado con su cuenta de Twitter y reflejado en las guías por materias que muestran esta misma información de forma sincronizada (Archambault, 2012). http://libanswers.Imu.edu/a.php?qid=135211 https://twitter.com/Imulibrarianedu

\section{Servicios virtuales}

La mayoría de bibliotecas los ofrecen desde sus webs, sin embargo esta acción resulta insuficiente. No se trata sólo de poner enlaces al servicio, sino que se debería aumentar la información que le rodea, ampliando el texto y los gráficos para describirlo mejor, y colocando enlaces en sitios estratégicos con etiquetas más amigables. La mayoría de usuarios no saben a qué se refiere el logo del servicio, y la difusión debe hacerse tanto dentro como fuera de la biblioteca (Connaway; Radford, 2011).

En la Wisconsin University Library se ha añadido al catálogo un pop-up que se despliega ofreciendo ayuda del bibliotecario cuando no se encuentra lo que se busca (Mu et al., 2011).

Otra tendencia al alza en bibliotecas universitarias es la de 


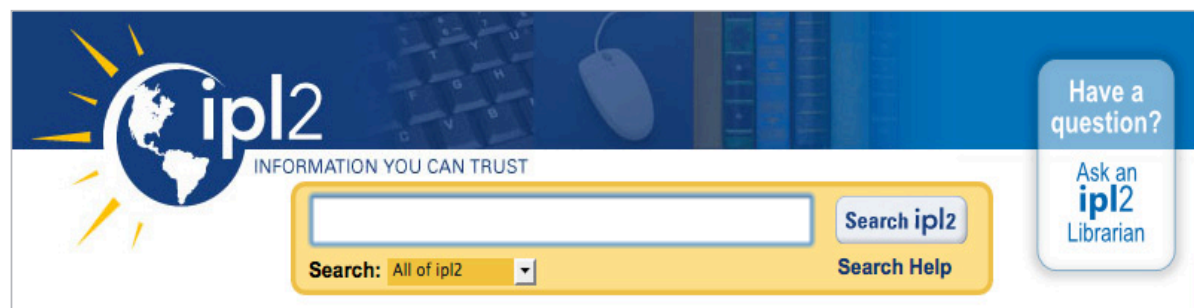

Spotlight: The Link: Back to School Edition

(1)
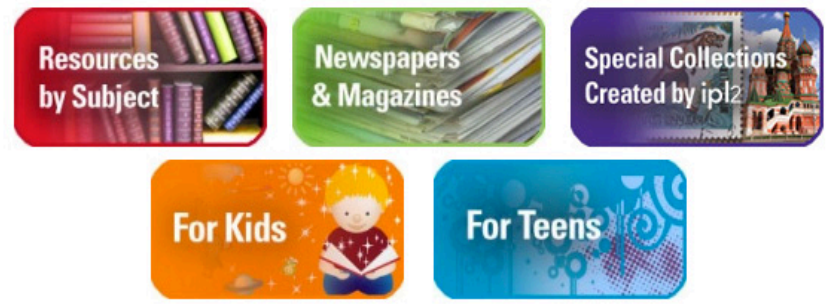

Connect With Us On 10 (3)

Home | About | Privacy Policy | Contact Us | Newsletter | Library Locator | Site Map | Ask an ipl2 Librarian Partners: Drexel| | FSU | Buffalo | |llinois | Pittsburgh | Rutgers | UNC $\mid$ Washington | Join the ip/2 Consortium

Imagen 3. Internet Public Library de la Michigan University

servicios colaborativos entre bibliotecas consorciadas o mediante acuerdos institucionales para compartir personal, recursos y horario de atención al público. Destacan Global Reference Network, integrada por centros de todo el mundo que emplean QuestionPoint como sistema para administrar su referencia digital, y la iniciativa Internet Public Library de la Michigan University, que cuenta con la colaboración de estudiantes y bibliotecarios voluntarios de todo el mundo, que responden a consultas sobre referencia.

http://www.ipl.org

En España sorprende que existiendo consorcios de bibliotecas no se ofrezca este servicio de forma consorciada (Manso-Rodríguez, 2010). Tan sólo encontramos la colaboración entre las bibliotecas públicas españolas y algunas universitarias con el programa QuestionPoint en el servicio Pregunte al bibliotecario (Merlo-Vega, 2009).

\section{Aplicaciones de la web social}

Cuando en el servicio de referencia se integran aplicaciones de la web social, podemos hablar de referencia 2.0. Ayra y Mishra (2011) realizaron un estudio sobre su uso en el servicio de referencia virtual, destacando el uso del chat, seguido a mucha distancia por Facebook o Twitter. Sin embargo y aunque las redes sociales favorecen el aumento de las transacciones de referencia, se encuentran muy poco integradas en este servicio, salvo casos excepcionales como las bibliotecas de la Western Washington University, y en líneas generales adolecen de falta de visibilidad y promoción, ya que los usuarios desconocen que la biblioteca los ha puesto para comunicarse con ellos (Ramos; Abrigo, 2012). Esta situación se mantiene también en el ámbito español, con presencia de bibliotecas en medios sociales, pero no de su servicio de referencia (Manso, 2010).

https://twitter.com/Ask_WWU_Lib

Las tecnologías sociales facilitan la participación y colaboración del usuario (Pinto; Manso, 2012), que llega a formar parte del servicio contribuyendo a su diseño, elaboración y contenidos, tendencia que constituye una de las bases del marketing 3.0. Esta versión del marketing se basa en la implicación del usuario en el proceso creativo -el llamado marketing de colaboración- con la idea de que los consumidores aprecien cada vez más la colaboración en el proceso de creación, desarrollo y comunicación de la marca (Kotler et al., 2012).

La referencia social, muy ligada a la referencia 2.0, aparece cuando una comunidad de voluntarios responde a las preguntas de otros usuarios, e implica un notable esfuerzo de colaboración en grupo (Shachaf, 2010). Un servicio similar es el chat de Twitter usado mediante un \#hashtag, a través del cual se puede seguir una conversación y participar sobre

un tema. Un ejemplo de referencia virtual 2.0 y social es Bibliosesame, una red nacional de bibliotecas públicas que responden de forma colaborativa. En su página en Facebook los usuarios plantean preguntas en su muro que son respondidas por bibliotecarios de la red y cualquier usuario puede participar y enriquecer las respuestas.

https://www.facebook.com/bibliosesame

Second Life fue uno de los primeros servicios de la web social en el que los bibliotecarios ofrecieron la referencia virtual y supone una oportunidad para aquellas bibliotecas en cuyas universidades se está impartiendo docencia en estos espacios virtuales, ya que puede de esta forma interactuar con sus usuarios y responder a sus preguntas en los espacios que frecuentan (Godfrey, 2008).

Se deben integrar los planes de marketing en los planes estratégicos

La realidad aumentada (RA), es otra de las tendencias tecnológicas que puede tener mayor utilidad integrada en los servicios de referencia. Consiste en superponer información a los objetos que tenemos delante visualizados a través de una cámara. Se puede aplicar para muchas de las preguntas que plantean los usuarios de las bibliotecas universitarias: preguntas de respuesta rápida, información básica sobre la organización de la biblioteca, localización de materiales, etc. La integración de la RA en los folletos, guías o impresos de la biblioteca amplía el servicio de referencia. Una de las aplicaciones más divulgadas en bibliotecas universitarias es WolfWalk, de la North Carolina State University, que ofrece imágenes históricas de los archivos de la biblioteca en más de 50 puntos a lo largo del campus, además de información básica sobre la biblioteca (Bishop; Bartlett, 2012).

La referencia móvil se encuentra entre las 10 tendencias para las bibliotecas universitarias que la ACRL publicaba este año 2012 (Andersen; Russell, 2012). Se destaca el en- 
torno móvil ya que está cambiando la forma en la que se accede a la información, y es la vía preferida de muchos para el acceso al servicio de referencia (Accart, 2012).

Los dispositivos móviles se usan también para la referencia en movilidad y para la lectura de los códigos QR ubicados en la biblioteca para ayudar a los usuarios a localizar materiales, servicios y salas (Henry et al., 2012). Se convierten en una potencial solución para el servicio de referencia, con la creación de aplicaciones específicas (apps) de la biblioteca, para que los usuarios encuentren las respuestas a sus preguntas (Bishop, 2012).

La inteligencia artificial también se aplica a estos servicios mediante los agentes de respuesta automática. Son ejemplos Pixel en la University of Nebraska-Lincoln Libraries y Stela, de la Hamburg University Library, un robot experimental que puede responder a preguntas sobre la biblioteca y sus recursos, con la mímica de una conversación humana. http://pixel.unl.edu

http://www.sub.uni-hamburg.de/bibliotheken/projekte/ chatbot-stella.html

Un ejemplo en España de inteligencia artificial es el servicio 24 h. Pregunta al bibliotecario de la Biblioteca de la Universidad de Jaén, que funciona 24/7 facilitando una respuesta rápida a las necesidades de información.

\section{Bibliotecarios integrados}

El bibliotecario personal no es una nueva forma de ofrecer el servicio de referencia, pero cobra protagonismo cuando se transforman los mostradores de referencia, y se potencia la figura del bibliotecario temático.

Para Kilzer (2011), el bibliotecario universitario debe integrarse en la infraestructura del campus y tener agilidad para responder a las necesidades que surjan. En la Texas Tech University, el bibliotecario temático se presenta a los estudiantes como su bibliotecario personal, su persona de contacto en el campus, y publica vídeos de utilidad para toda la comunidad universitaria. La actividad del servicio tras esta campaña aumentó un $120 \%$ en sólo dos años. También en las universidades Yale y Drexel se asigna un bibliotecario personal a cada estudiante.

Con estos nuevos roles desaparece la privacidad y anonimato de la biblioteconomía tradicional, ya que gana en visibilidad y, como dicen Mon y Harris (2012), es difícil ser valorado cuando se es invisible. Además de ganar visibilidad, el servicio debe personalizarse, y para ello es importante que el bibliotecario pueda ser identificado físicamente y en su web (véase el ejemplo de las bibliotecas de la Colorado Boulder University). Mon y Harris (2012) se plantean si la cultura de la organización estará dispuesta a este necesario cambio.

\section{http://ucblibraries.colorado.edu/askus/index.htm}

Por último, el bibliotecario embebido o integrado, aparece continuamente en la literatura profesional y se utiliza para describir el trabajo de un bibliotecario universitario que participa de forma permanente, presencial o virtual, en la formación en competencias informacionales integrada en los currículos o en las reuniones departamentales, y sobre todo como apoyo a los investigadores. Para Torres-Salinas (2012)

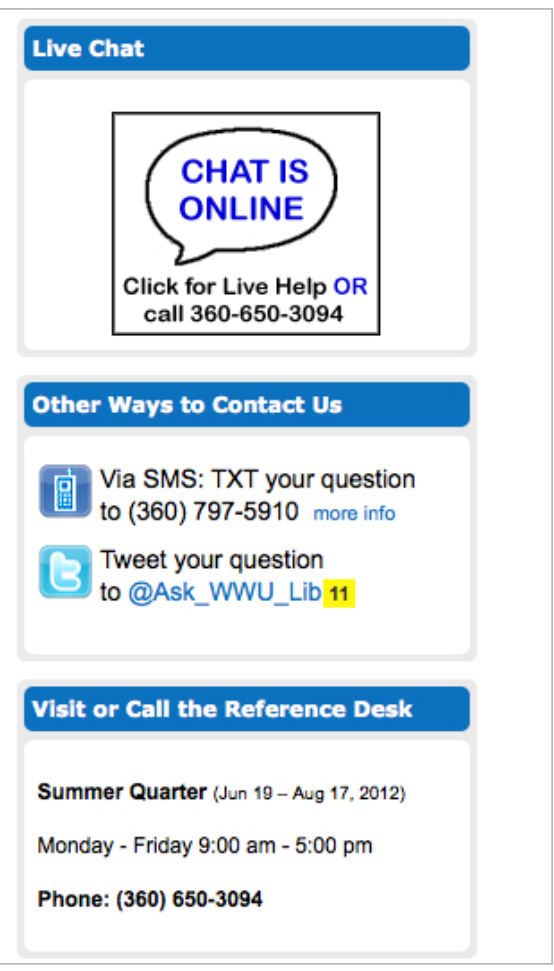

Imagen 4. Servicio Askus en WWU con Twitter http://askus.library.wwu.edu

estamos hablamos de un bibliotecario al que se le añaden nuevas competencias, que se encuentra especializado en un área temática, y que se identifica con grupos de trabajo, que sale fuera de los muros de la biblioteca y de los límites tradicionales de su profesión, integrándose en los despachos, y llevando a cabo tareas que antes sólo llevaba a cabo el profesor o el investigador.

En este sentido hay ejemplos muy significativos como el de la Biblioteca de la Michigan University (Alexander et al., 2011), donde se creó la figura del field librarian, o bibliotecario conectado con un departamento en concreto más que con una colección física y los bibliotecarios de apoyo a líneas de investigación.

Los bibliotecarios universitarios están transformando su servicio de referencia aumentando la visibilidad y accesibilidad del mismo, dentro y fuera de los espacios de la biblioteca, utilizando herramientas colaborativas de la web social, colaborando con profesionales y adoptando una actitud más proactiva, adelantándose y preguntándole al usuario por sus necesidades de información, e identificándose con grupos y líneas de investigación.

Para que todas estas nuevas iniciativas y cualquier otra forma tradicional de referencia tengan éxito, es imprescindible la evaluación continua del servicio, recogiendo datos sobre la actividad que se desarrolla, estableciendo indicadores que permitan medir su evolución y comprobando si se han alcanzado los objetivos propuestos. Este proceso es necesario no sólo como forma de control sino también para poner en evidencia el trabajo que se realiza. Se hace pues necesario el establecimiento de un plan de marketing (Andersen; Russell, 2012) en el que se definan las acciones que se deben llevar a cabo para conseguir el objetivo marcado, que en definitiva será un mayor uso del servicio de referencia. 


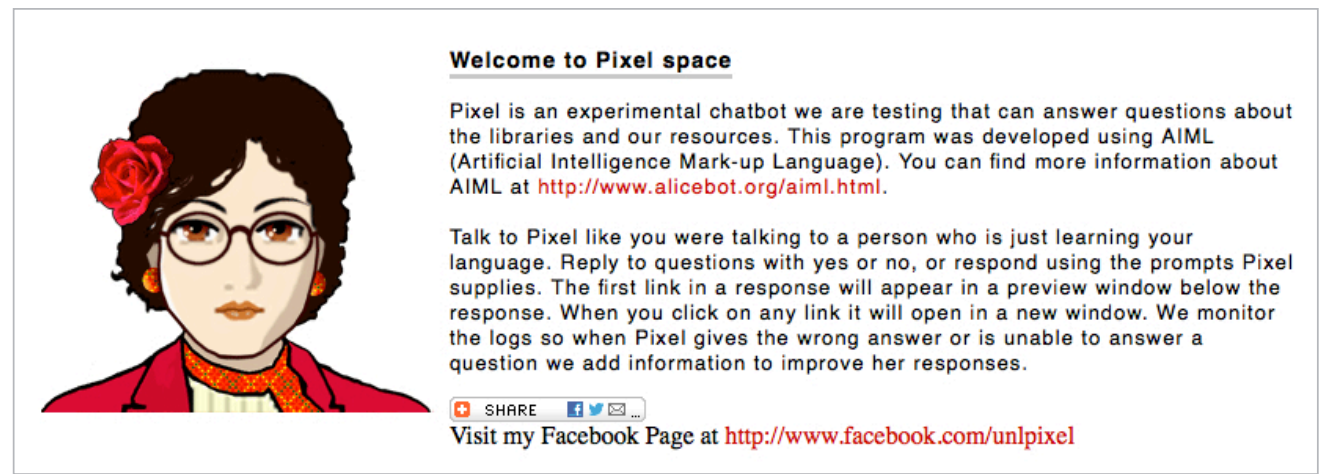

Imagen 5. Robot Pixel en la UNL

\section{Plan de marketing}

Las bibliotecas universitarias se esmeran en la elaboración de sus planes estratégicos, pero no se han preocupado suficientemente de implantar planes de marketing (GonzálezFernández-Villavicencio, 2009). Las posibilidades de conseguir los objetivos que se fijan en los planes estratégicos dependen en gran medida de la existencia de un plan de marketing integrado en el estratégico (Germano; StretchStephenson, 2012). Con el marketing no sólo se obtienen datos sino también información compleja y cualitativa sobre el comportamiento del usuario, sus comentarios, y las posibilidades de interacción y de retención como clientes, por lo que no contar con estos datos produce un vacío entre la oferta del servicio de biblioteca y lo que el usuario realmente necesita. El análisis de los datos puede evidenciar una necesidad de cambio o de reconfiguración de los servicios y los recursos, para que estén en consonancia con las necesidades de los usuarios y la creación de valor.

Antes de lanzar un nuevo servicio o producto hay que conocer las expectativas del usuario, es decir, el bien que los usuarios piensan obtener del mismo (Hsieh; Yuan, 2010) para que se convierta en una auténtica experiencia de usuario.

Entre los pasos que debemos dar a la hora de establecer un plan de marketing se encuentran los siguientes:

1. Definir el objetivo específico que se quiere obtener con el servicio de referencia. La escucha en los medios sociales puede ayudar a que responda a una necesidad de los usuarios que podamos satisfacer. El objetivo debe ser además medible y cuantificable, para establecer métricas que permitan evaluar si se está logrando.

2. Seleccionar el segmento al que nos vamos a dirigir, el público objetivo. En la Mississippi State University llevaron a cabo estudios para identificar el tipo de usuario que más utilizaba cada modalidad de sus servicios de referencia, para dirigirse específicamente a cada uno de ellos, adecuarse mejor a sus necesidades e identificar los motivos por los que no lo usaban (Nolen et al., 2012). Mitchell et al. (2011) describen cómo seleccionaron su público objetivo, los estudiantes de nuevo ingreso, para después establecer todas las actuaciones que se llevarían a cabo para atraerlos hacia el servicio de referencia, desde acciones directas, hasta acuerdos con los profesores, y demás servicios de la universidad.

3. Establecer los espacios o canales que vamos a utilizar. En la Michigan University Library el servicio de referencia tiene desde enero de 2010 una sección fija en la web de noticias de la Universidad, dedicada especialmente a profesores y personal investigador (Alexander et al., 2011). Los medios sociales son un canal perfecto para la promoción y oferta de los servicios de referencia, y algunas bibliotecas universitarias utilizan Facebook y Twitter para promocionar los servicios, con resultados muy positivos (Ramos; Abrigo, 2012). Sin embargo, no se trata de estar en todos los medios sociales sino en aquellos que se adecuen mejor al objetivo y público destinatario del servicio.

\section{http://ur.umich.edu/update}

4. El plan de acción señala las acciones necesarias para conseguir los objetivos. Una etapa crucial de ese plan es la evaluación y medición de los resultados, ya que es la única forma de saber si se está logrando el objetivo y la eficacia del servicio. No es infrecuente una cierta reticencia a la obtención de estos datos por parte del personal (Logan; Lewis, 2011). Otro de los errores más comunes es creer que el único servicio que hay que evaluar es el último que se implanta, y que en muchos casos consiste en la versión virtual de un servicio tradicional. Los requisitos que se le exigen a la versión virtual no suelen coincidir con los demandados para la versión tradicional.

\section{Las bibliotecas universitarias no han rea- lizado suficiente marketing de sus servicios}

\section{Evaluación}

La evaluación del servicio de referencia según Pomerantz et al. (2008), debe realizarse desde la perspectiva tanto del servicio, horas, materiales, calidad de las respuestas (el valor implícito), como del usuario: grado de satisfacción, compromiso y difusión por su parte (valor explícito).

Los programas utilizados para recoger datos y elaborar estadísticas de referencia son en la mayoría de los casos de reciente aparición, ya que el método más usado, cuando existe alguno, es la hoja de cálculo. Algunos de estos programas son Libstats (Queensland University), Desk Tracker (Illinois University), LibAnswers (Western University Libraries), Gimlet (Loyola Marymount University), KnowBot (Northwest Missouri State University), o incluso un formulario en Google Docs (Minnesota University).

http://www.infotoday.com/cilmag/oct11/Carter_Ambrosi. shtml

Para el análisis de los datos se utilizan programas estadísticos como SPSS o módulos de estadística incluidos en los mismos programas. Cuando lo que se quiere medir es el 
grado de satisfacción del usuario, se utiliza LibQUAL+. Otros métodos usados desde este mismo punto de vista son el buzón de sugerencias, la observación (directa o indirecta), las encuestas y entrevistas, los grupos focales y los estudios de caso (Cassell; Hiremath, 2011).

La referencia virtual tiene una gran ventaja sobre la presencial y es la posibilidad de analizar las transacciones, que aportan información de gran valor tanto de tipo estadístico como desde la perspectiva del usuario (Bishop, 2012). Mediante el estudio de las transacciones la biblioteca puede optimizar su funcionamiento. Tras el análisis de las preguntas y respuestas del servicio de referencia virtual con LibraryH3lp, en la Cardiff University los bibliotecarios observaron que los usuarios demandaban la ampliación del servicio en horario de tarde, noche y fines de semana (Haerkoenen et al., 2011).

No se trata de esperar que el usuario venga sino de salir a su encuentro, innovar y sorprender

Como ejemplo de evaluación del servicio en su conjunto presencial y virtual- la Biblioteca de la Universidad de Sevilla (BUS), tras la puesta en marcha del servicio en línea (chat con LibraryH3/p) en 2009 (González-Fernández-Villavicencio et al., 2009), procedió al análisis conjunto del servicio. Para ello se realizó en 2011 un diagnóstico de la situación en todas las bibliotecas de área a partir de la toma de datos de las preguntas de información y referencia que los usuarios realizaban a los bibliotecarios de la BUS, y durante un período de tiempo establecido. Para el análisis del servicio se adoptó el modelo Read (Reference effort assessment data), que intenta registrar el esfuerzo y nivel de conocimiento del bibliotecario en cualquier consulta, al que se añadieron aspectos como número de preguntas, tipo, patrones de comportamiento, nivel de satisfacción de los usuarios y formación adecuada del personal. En el estudio posterior de los datos se analizó el tema y dificultad de las consultas, las respuestas y su duración, tipos de personal bibliotecario y de los usuarios, horarios y medio de comunicación utilizado. La abrumadora cantidad de datos obtenidos con esta evaluación y el análisis posterior realizado por un equipo de trabajo de la biblioteca, recomendaron actuaciones no sólo sobre el servicio de referencia en su doble modalidad presencial y virtual, sino también sobre la organización y el resto de los servicios de la biblioteca.

Por último, como resultado del plan de marketing es preciso ofrecer un producto que sea una auténtica experiencia de usuario, que lo tenga en cuenta y lo involucre. No se trata sólo de atender su consulta, sino de hacer que el usuario sea partícipe del servicio (Connaway; Radford, 2011).

\section{Conclusiones}

Se han presentado diferentes tendencias en los servicios de referencia en todo el mundo, a partir de las cuales se proponen las siguientes líneas de trabajo:

- Potenciar y fomentar una actitud proactiva que lleve a salir fuera de los muros de la biblioteca, incluso de los espacios de la universidad, con servicios de referencia móvil que vayan donde esté el posible usuario. Se trata de no esperar que el usuario venga sino salir a su encuentro, innovar y sorprender.

- Incorporar características de la web social y móvil al servicio de referencia, siendo conscientes de que los usuarios están en los medios sociales y que el futuro de la referencia será principalmente móvil y social.

- Colaborar con usuarios y profesionales, universitarios y no universitarios (Cmor, 2010), estudiantes cualificados, especialistas TIC y otros servicios de la universidad, para centrar la actividad de referencia en el usuario y ser fieles al espíritu crai.

- Fortalecer la figura del bibliotecario temático y su integración en los departamentos y en los proyectos de investigación.

- Diseñar planes de marketing que promocionen el servicio de referencia y mejoren su visibilidad, e integren al usuario en el diseño del proceso creativo del servicio (marketing 3.0).

- Evaluar y mostrar datos que evidencien el éxito del servicio, en la medida en la que contribuye a los objetivos de la academia. Evidenciar la utilidad es básico para que los órganos rectores de la universidad atiendan las demandas de recursos de la biblioteca.

El futuro de la referencia es multimodal y hay que ofrecer una gran variedad de servicios según las necesidades de las cambiantes audiencias $y$ las preferencias de los usuarios (Kilzer, 2011). No hay que olvidar que estamos en la época del consumo basado en situaciones: diferentes productos cubren las distintas necesidades de información de los usuarios. El servicio de referencia de la biblioteca universitaria tiene que estar presente en cualquier lugar y a cualquier hora, disponible para cualquier persona, por el mayor número de canales (Nolen et al., 2012).

En el caso de que los resultados no sean positivos, no se debe entrar en el círculo vicioso de reducir los servicios. Si éstos no se usan algo se está haciendo mal. Es evidente que no se está ofreciendo lo que

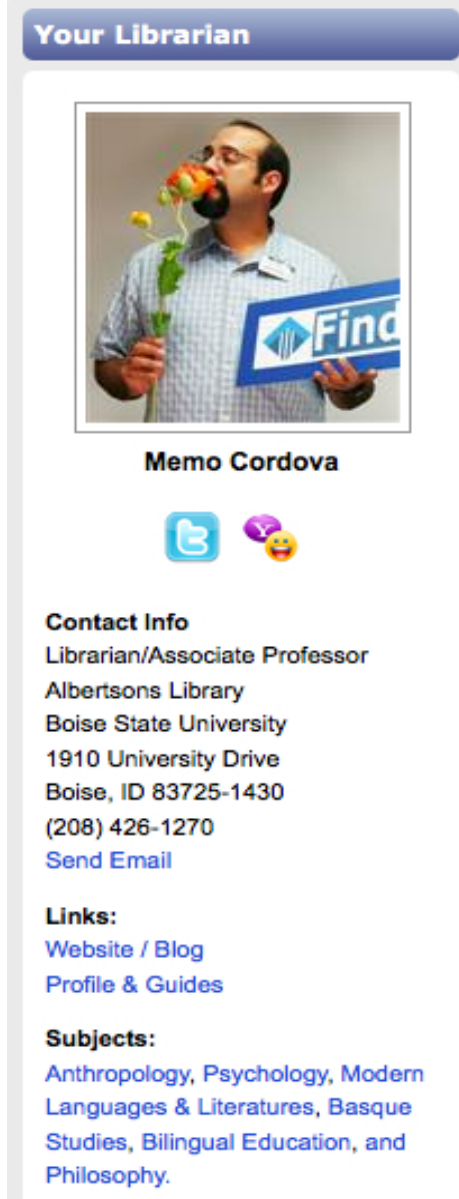

Imagen 6. Bibliotecario de referencia en la Boise State University

http://guides.boisestate.edu/profile. php?uid $=2184$ 


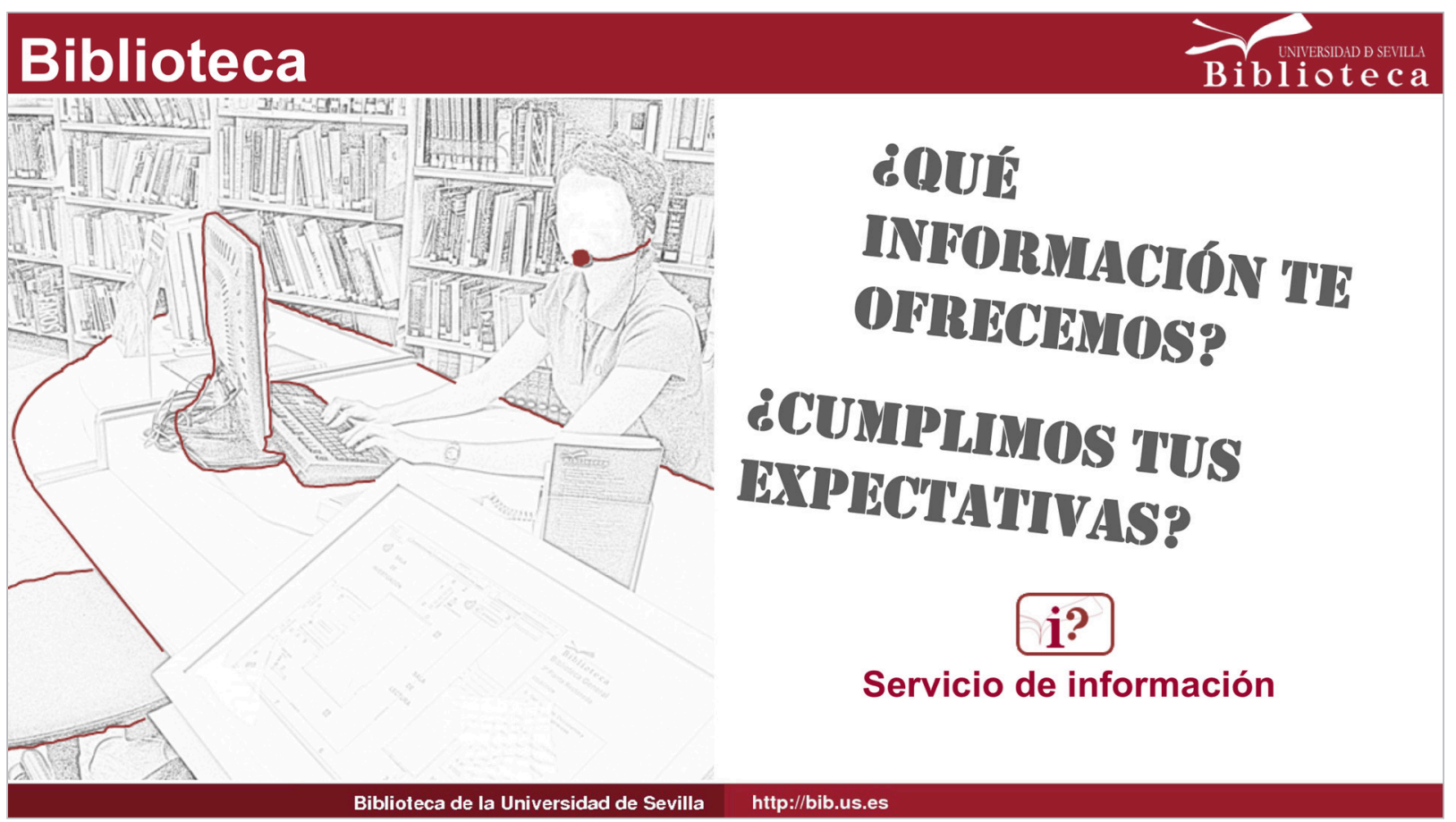

Imagen 7. Difusión del período de toma de datos para la evaluación del servicio de referencia en la BUS

necesitan los usuarios dónde y cuándo lo necesitan, no se evalúan los resultados ni se adoptan medidas correctoras en función de esos datos, no se aplican planes de marketing que optimicen el servicio y los hagan visibles, personalizados, móviles y sociales...

Por otro lado las bibliotecas universitarias deben demostrar el valor que aportan a su institución, los estudiantes, los docentes, los investigadores y la comunidad en general (Oakleaf, 2010), considerando su impacto en la matriculación (Lombard, 2012), retención de estudiantes (Haddow; Joseph, 2012) y en los resultados de su aprendizaje, el éxito en sus estudios, en sus logros, en su experiencia, o en la retención de los profesores y número de sus publicaciones en revistas de impacto (Neal, 2011). Investigaciones recientes se están centrando en la correlación entre el impacto de los servicios de referencia y apoyo a la docencia de los bibliotecarios y los logros académicos de los estudiantes y profesores universitarios (Cooke et al., 2011).

Hay que diseñar planes de marketing que promocionen el servicio de referencia, mejoren su visibilidad e integren a los usuarios

La organización y el suministro de la información requieren hoy una aproximación más emprendedora, personalizada y orientada al marketing (Nolen et al., 2012). Para Miller (2012) las bibliotecas universitarias no pueden seguir pensando en un futuro lineal, sino en uno de continua transformación. Se necesitan líderes visionarios, no simplemente gestores.

\section{Bibliografía}

Accart, Jean-Philippe. "Hot off the press: digital reading" Library hi tech news, 2012, v. 29, n. 7.

Alexander, Laurie; Blumenthal, Jane; Downing, Karen; MacAdam, Barbara; Rana, Gurpreet K.; Reiman-Sendi, Karen; Scholtz, Nicole; Sutch, Laurie A. "Mlibrary: concepts for redefining reference". Journal of library administration, 2011, v. 51, n. 4, pp. 326-342.

http://dx.doi.org/10.1080/01930826.2011.556957

Andersen, Laura; Russell, Beth. "2012 top ten trends in academic libraries: a review of the trends and issues affecting academic libraries in higher education". College and research libraries news, v. 73, n. 6, pp. 311-320.

http://crln.acrl.org/content/73/6/311.full?etoc

Archambault, Susan-Gardner. "Desk statistics under a microscope = Improved library services". En: 78 IFLA General conference and assembly, 2012, pp. 1-22.

http://conference.ifla.org/sites/default/files/files/papers/ wlic2012/203-archambault-en.pdf

Arndt, Theresa S. "Reference service without the desk". Reference services review, 2010, v. 38, n. 1, pp. 71-80. http://dx.doi.org/10.1108/00907321011020734

Arya, Harsh Bardhan; Mishra, J. K. "Oh! Web 2.0, virtual reference service 2.0 , tools \& techniques (I): a basic approach". Journal of library \& information services in distance learning, 2011, v. 5, n. 4, pp. 149-171.

http://www.tandfonline.com/doi/pdf/10.1080/153329 ox.2011.638819

http://dx.doi.org/10.1080/1533290X.2011.638819

Attis, David; Koproske, Colin; Miller, Chris; Matovich, Kevin; Tisdale, Hillary. Redefining the academic library. Managing the migration to digital information services. Washing- 
ton, DC, The Advisory Board Company, 2011.

Bell, Steven J. "Who needs a reference desk?" Library issues, 2007, v. 27, n. 6.

Bishop, Bradley Wade. "Analysis of reference transactions to inform library applications (apps)". Library and information science research, 2012, v. 34, n. 4, pp. 265-270. http://dx.doi.org/10.1016/j.lisr.2012.06.001

Bishop, Bradley-Wade; Bartlett, Jennifer. "Where do we go from here?: informing academic library staffing through reference transaction analysis". College and research libraries, pre-print, 2012.

Cassell, Kay-Ann; Hiremath, Uma. Reference information services in the 21st century: an introduction. (2nd edition revised). London: Neal-Schuman Publishers, 2011. ISBN: 978 1555707408

Cmor, Dianne. "Academic reference librarians: getting by with a little help from our (special, public, school, law and medical librarian) friends". Library management, 2010, v. 31, n. 8/9, pp. 610-620.

http://dx.doi.org/10.1108/01435121011093388

Coffman, Steve. "The decline and fall of the library empire". Information today, v. 20, n. 3, 2012.

http://www.infotoday.com/searcher/apr12/Coffman--TheDecline-and-Fall-of-the-Library-Empire.shtml

Connaway, Lynn S.; Radford, Marie L. Seeking synchronicity: revelations and recommendations for virtual reference. Dublin, OH: OCLC Research, 2011.

http://www.oclc.org/reports/synchronicity/full.pdf

Cooke, Louise; Norris, Michael; Busby, Nial; Page, Thomas; Franklin, Ginny; Gadd, Elizabeth; Young, Helen. "Evaluating the impact of academic liaison librarians on their user community : a review and case study". New review of academic librarianship, 2011, Sept., v. 17, n. 1, pp. 5-30.

Dempsey, Megan. "Blending the trends: a holistic approach to reference services". Public services quarterly, 2011, v. 7, n. 1-2, pp. 3-17.

http://dx.doi.org/10.1080/15228959.2011.572769

Gadsby, Joanna; Qian, Shu. "Using an iPad to redefine roving reference service in an academic library". Library hi tech news, 2012, v. 29, n. 4, pp. 1-5.

http://dx.doi.org/10.1108/07419051211249446

Germano, Michael; Stretch-Stephenson, Shirley M. "Strategic value planning for libraries". The bottom line: managing library finances, 2012, v. 25, n. 2, pp. 71-88. http://dx.doi.org/10.1108/08880451211256405

Godfrey, Krista. "A new world for virtual reference". Library hi tech, 2008, v. 26, n. 4, pp. 525-539.

http://dx.doi.org/10.1108/07378830810920879

González-Fernández-Villavicencio, Nieves. "Bibliotecas y marketing en red". BiD: textos universitaris de biblioteconomia i documentació, 2009, desembre, n. 23.

http://www.ub.edu/bid/23/gonzalez2.htm

González-Fernández-Villavicencio, Nieves; Barrera-Gómez, Juan-Antonio; Gómez-Fernández, María-José; Moscoso-
Castillo, Miriam; Santos-Flores, Victoria; Suárez-Samaniego, Marta. "Referencia virtual en la biblioteca de la Universidad de Sevilla: una experiencia colectiva". El profesional de la información, 2009, v. 18, n. 6. pp. 633-641. http://dx.doi.org/10.3145/epi.2009.nov.06

Haddow, Gaby; Joseph, Jayanthi. "Loans, logins, and lasting the course: academic library use and student retention". Australian academic and research libraries, 2010, v. 41, n. 4, pp. 1-10.

http://alia.org.au/publishing/aarl/41/AARL_2010_41_4. $p d f$

Haerkoenen, Sonja; Blackmore, Andrew; Beadle, Rob. Creating a successful chat library service: ask a librarian live at Cardiff University, 2011, pp. 1-14 [copia suministrada por los autores].

Henry, C. Cynthia L.; Vardeman, Kimberly K.; Syma, Carrye K. "Reaching out: connecting students to their personal librarian". Reference services review, 2012, v. 40, n. 3, pp. 396-407.

http://dx.doi.org/10.1108/00907321211254661

Hsieh, Yen-Hao; Yuan, Soe-Tsyr. "Modeling service experience design processes with customer expectation management. A system dynamics perspective". Kybernetes, 2010, v. 39, n. 7 pp. 1128-1144.

http://dx.doi.org/10.1108/03684921011062746

Kilzer, Rebekah. "Reference as service, reference as place: a view of reference in the academic library". The reference librarian, 2011, v. 52, n. 4, pp. 291-299.

http://dx.doi.org/10.1080/02763877.2011.588539

Kotler, Philip; Kartajaya, Hermawan; Setiawan, Iwan. Marketing 3.0. Madrid [etc.]: LID, 2012.

Langan, Kathleen A. "Training millennials: a practical and theoretical approach". Reference services review, 2012, v. 40, n. 1, pp. 24-48.

http://dx.doi.org/10.1108/00907321211203612

[Libref-I]. Eliminating the physical reference desk, 2012, 22 May.

Logan, Firouzeh F. "A brief history of reference assessment: no easy solutions". The reference librarian, 2009, v. 50, n. 3, pp. 225-233.

http://dx.doi.org/10.1080/02763870902947133

Logan, Firouzeh F.; Lewis, Krystal. "Quality control: a necessary good for improving service". The reference librarian, 2011 , v. 52 , n. 3 , pp. $218-230$.

http://dx.doi.org/10.1080/02763877.2011.557314

Lombard, Emmett. "The role of the academic library in college choice". The journal of academic librarianship, 2012, v. 38, n. 4, pp. 237-241.

http://dx.doi.org/10.1016/j.acalib.2012.04.001

Luini, Christina. Virtual reference service: a case study of QuestionPoint utilization at the Gallagher Law Library. Seattle, Washington: University of Washington Information School, 2012.

http://lib.law.washington.edu/lawlibrarianship/ CILLPapers/Luini2012.pdf 
Manso-Rodríguez, Ramón-Alberto. Servicio de referencia virtual: propuesta de un modelo basado en criterios de calidad y herramientas de la web 2.0. Tesis doctoral, Universidad de Granada, 2010.

http://hera.ugr.es/tesisugr/19562871.pdf

Merlo-Vega, José-Antonio. "Referencia digital: concepto, tecnologías e implementación en centros de información". El profesional de la información, 2009, v. 18, n. 6, pp. 589600.

http://gredos.usal.es/jspui/bitstream/10366/70659/3/ DBD_Referencia_digital.pdf

http://dx.doi.org/10.3145/epi.2009.nov.02

Miller, Rush. "Damn the recession, full speed ahead". Journal of library administration, 2012, v. 52, n. 1, pp. 3-17.

http://www.acrl.ala.org/ULS/?p=481

Mitchell, Megan S.; Comer, Cynthia H.; Starkey, Jennifer M.; Francis, Eboni A. "Paradigm shift in reference services at the Oberlin College Library: a case study". Journal of library administration, 2011, v. 51, n. 4 pp. 359-374.

http://dx.doi.org/10.1080/01930826.2011.556959

Mon, Lorri; Harris, Lydia-Eato. "The death of the anonymous librarian". The reference librarian, 2011, v. 52, n. 4, pp. 352-364.

http://dx.doi.org/10.1080/02763877.2011.585279

Mu, Xiangming; Dimitroff, Alexandra; Jordan, Jeanette; Burclaff, Natalie. "A survey and empirical study of virtual reference service in academic libraries". The journal of academic librarianship, 2011, v. 37, n. 3, pp. 120-129.

http://dx.doi.org/10.1016/j.acalib.2011.02.003

Neal, James G. "Stop the madness: the insanity of ROI and the need for new qualitative measures of academic library success". En: ACRL, Philadelphia, Pennsylvania, 2011, pp. 424-429.

http://www.ala.org/acrl/sites/ala.org.acrl/files/content/ conferences/confsandpreconfs/national/2011/papers/ stop_the_madness.pdf

Nolen, David S.; Powers, Amanda-Clay; Zhang, Li; Xu, Yue; Cannady, Rachel E.; Li, Judy. "Moving beyond assumptions: the use of virtual reference data in an academic library". Portal: libraries and the academy, 2012, v. 12, n. 1, pp. 23-40. http://www.press.jhu.edu/journals/portal_libraries_and_ the_academy/portal_pre_print/current/articles/12.1nolen. $p d f$

Oakleaf, Megan. ACRL. The value of academic libraries. ACRL Asociation of College and Research Libraries, 2010.

http://www.acrl.ala.org/value
Penner, Katherine. "Mobile technologies and roving reference". Public services quarterly, 2011, v. 7, n. 1-2, pp. 27-33. http://dx.doi.org/10.1080/15228959.2011.572775

Pinto, María; Manso, Ramón-Alberto. "Virtual references services: defining the criteria and indicators to evaluate them". The electronic library, 2012, v. 30, n. 1, pp. 51-69. http://dx.doi.org/10.1108/02640471211204060

Pomerantz, Jeffrey; Mon, Lorri; McClure, Charles R. "Evaluating remote reference service: a practical guide to problems and solutions". Portal: libraries and the academy, 2008 , v. 8, n. 1, pp. $15-30$.

http://mcclure.ii.fsu.edu/publications/2008/Evaluating\%20 remote\%20reference\%20service.pdf

Ramos, Marian S.; Abrigo, Christine M. "Reference 2.0 in action: an evaluation of the digital reference services in selected Philippine academic libraries". Library hi tech news, 2012 , v. 29, n. 1, pp. 8-20.

http://dx.doi.org/10.1108/07419051211223426

RUSA. Definitions of reference, 2008.

http://www.ala.org/rusa/resources/guidelines/definitionsreference

Saunders, Laura. "The reality of reference: responsibilities and competencies for current reference librarians". Public services quarterly, 2012, v. 8, n. 2, pp. 114-135.

http://dx.doi.org/10.1080/15228959.2012.662074

Schulte, Stephanie J. "Eliminating traditional reference services in an academic health sciences library: a case study". Journal of the Medical Library Association: JMLA, 2011, v. 99, n. 4, pp. 273-279.

http://dx.doi.org/10.3163/1536-5050.99.4.004

Shachaf, Pnina. "Social reference: toward a unifying theory". Library and information science research, 2010, v. 32, n. 1, pp. 66-76.

http://dx.doi.org/10.1016/j.lisr.2009.07.009

Torres-Salinas, Daniel. "Incrustados e integrados en la investigación: los embedded librarians". Anuario ThinkEPI, 2010, v. 5, pp. 48-51.

Wang, Bing; Henson, Bruce. "Change is coming: a combined services area project". Science and technology libraries, 2011, v. 30, n. 1, pp. 89-98.

http://dx.doi.org/10.1080/0194262X.2011.545678

Ward, David; Phetteplace, Eric. "Staffing by design: a methodology for staffing reference". Public services quarterly, 2012, Sept., v. 8, n. 3, pp. 37-41.

http://dx.doi.org/10.1080/15228959.2011.621856

\section{Suscripción EPI sólo online}

Pensando sobre todo en los posibles suscriptores latinoamericanos, ya no es obligatorio pagar la suscripción impresa de EPI para acceder a la online.

EPI se ofrece a instituciones en suscripción "sólo online" a un precio considerablemente más reducido (96,19 + 21\% IVA euros/año), puesto que en esta modalidad no hay que cubrir los gastos de imprenta ni de correo postal. 